Classification

Physics Abstracts

$61.50-61.55-81.40$

\title{
Double shear mechanism and orientation relationships in austenite/ferrite steels
}

\author{
Patricıa Donnadieu ( ${ }^{1}$ ) and Michel Duneau ( ${ }^{2}$ ) \\ ( $\left.{ }^{1}\right)$ Laboratoire d'Etude des microstructures $(*)$, BP 72, 92322 Châtillon Cedex, France \\ (2) Centre de Physique Théorique $\left({ }^{* *}\right)$, Ecole Polytechnique, 91128 Palaiseau Cedex, France
}

(Received 29 October 1993, accepted 1 December 1993)

\begin{abstract}
We propose a new approach of structural transformation between lattices by means of simple bounded transformations composed of two « fractional shears ». The strength point of this double shear mechanısm in lattice transformations is to specify particular orientation relationships. Though the basis of the model concerns the general 3D diffusionless transformation, the case of 2D lattices is presented in detail. When applied to the transformation of fcc (111) plane into the bcc (110) plane, the model is able to predict precise orientation relationships close to those observed in metallurgy (Kurdjumov-Sachs or Nishiyama-Wasserman relationships). This 2D model seems to be particularly relevant for materials such as the duplex steels.
\end{abstract}

\section{Introduction.}

In previous studies, the problem of mapping two different lattices onto each other by bounded transformations has been considered in the most general cases ( $n$-dimensional lattices) $[1,2]$. It was shown that any $n$-dimensional lattice can be mapped onto another lattice by a sequence of at most $n+1$ shear transformations provided that the two lattices have the same density of nodes. This property is preserved when fractional shears, bounded versions of the linear shear transformations, are considered. These bounded transformations define globally the displacement of each lattice point. Still, using the fractional shear transformation imposes that the shear vectors belong to the lattice.

Moreover, it was demonstrated that in some cases, less that $n+1$ shear operations are sufficient. In particular, two successive shears might be enough to map a lattice onto another one in 3D. The general case of $n+1$ shears is met when the two lattices have no particular orientation relationship. On the contrary, the transformation can be achieved by less than $n+1$ shears provided that the lattices exhibit particular orientation relationships.

(*) CNRS - UMR 104.

(**) CNRS - UPRA 0014. 
These bounded transformations are then meeting the main features of the martensitic transformations [3] : structural transformations between lattices without diffusion and existence of definite orientation relationships. We thus propose to examine in more detail the twodimensional case and consider whether it might describe some known metallurgical situation. We must underlıne first that double shear mechanisms have already been proposed for martensitic transformations but these models are macroscopic or phenomenological. In the present case by means of an exact model, we predict a set of orientation relationships which are in agreement with the observations.

In section 2, before specifying the two-dimensional (2D) case, we will recall some basic features concerning fractional shears and the double shear restriction is examined in section 3 . In section 4 , the orientation relationships between $2 \mathrm{D}$-lattices will be derived as function of the shear vectors. We give explicit solutions of the double shear mechanism which transform the $(110)_{b c c} 2 \mathrm{D}$-lattice into a $(111)_{f c c} 2 \mathrm{D}$ lattice in section 5 . The non-equivalent shear vectors (direction and amplutude) are given together with the corresponding orientation relationship. In section 6 , the predicted orientations will be compared with that encountered in martensitic transformations. The case of duplex steels will be considered with special interest since these materials might justify $2 \mathrm{D}$ model.

\section{Shears and fractional shears.}

This section recalls the geometrical definition of shear transformations and of fractional shears, their bounded versions. The latter transformation is of crucial importance. Without this bounded version the double shear mechanism developed in this paper would have no physical interest in the description of diffusionless transformations.

A shear is a linear mapping which transforms points by translations of fixed given direction. Using the notation of Dirac's brackets a 3D shear has the following form:

$$
S=I+|s\rangle\langle\sigma|=\left[\begin{array}{ccc}
1+s_{1} \sigma_{1} & s_{1} \sigma_{2} & s_{1} \sigma_{3} \\
s_{2} \sigma_{1} & 1+s_{2} \sigma_{2} & s_{2} \sigma_{3} \\
s_{3} \sigma_{1} & s_{3} \sigma_{2} & 1+s_{3} \sigma_{3}
\end{array}\right]
$$

so that the transformation reads :

$$
S(x)=x+\langle\sigma \mid x\rangle s .
$$

The vector $s$ determines the direction of the displacement. The vector $\sigma$, in reciprocal space, specifies the amplitude of the transformation and defines the invariant plane of the shear for $S(x)=x$ is equivalent to $\langle\sigma \mid x\rangle=0$. We shall consider shears of unit determinant, a condition equivalent to $\langle\sigma \mid s\rangle=0$ (the shear direction lies in the invariant plane and $s$ is invariant). In this case the inverse $S^{-1}$ is also a shear transformation given by:

$$
S^{1}=I-|s\rangle\langle\sigma|
$$

A shear transformation maps any lattice $\mathrm{L}$ onto a new lattice $\mathrm{L}^{\prime}=S(\mathrm{~L})$. The mapping $S$ is linear and therefore unbounded in the sense that $S(x)-x$ diverges when $x$ runs over $L$. In other words, the atomic displacements are not bounded so that this transformation is not a convenient model for a global diffusionless transformation between two lattices. However, if $s$ is a lattice vector of $\mathrm{L}$, and only in this case, the above shear transformation has a bounded version, called a fractional shear [1,2], which also transforms $L$ into $L^{\prime}$. If $S=I+|s\rangle\langle\sigma|$ is the linear shear, the associated fractional shear $\Sigma$ is defined by:

$$
\Sigma(x)=x+\operatorname{Frac}[\langle\sigma \mid x\rangle] s
$$


where Frac $[t]$ denotes the fractional part of the real number $t$, between $-1 / 2$ and $1 / 2$, so that $t=$ Round $[t]+$ Frac $[t]$ and Round $[t]$ denotes the nearest integer.

The displacement field $u(x)=\Sigma(x)-x=$ Frac $[\langle\sigma \mid x\rangle] s$ is now bounded by $\|s\| / 2$ so that $\Sigma$ is a bounded transformation. This field has the structure of a periodic modulation of wave vector $\sigma$, though the amplitude is not the usual Cosine or Sine function but the Frac function (see Fig. 1). Actually, $u(x)$ has the following Fourier expansion

$$
u(x)=\sum_{n \neq 0} \frac{(-1)^{n+1}}{2 \pi n} \sin (2 \pi n\langle\sigma \mid x\rangle) s .
$$

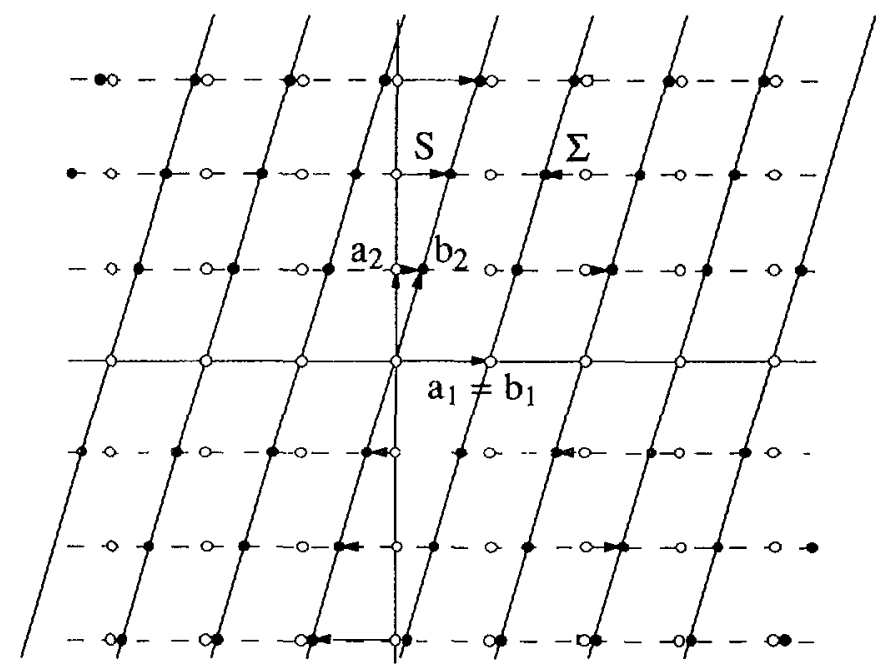

Fig. 1. - The linear shear $S$ has the lattice shear vector $a_{1}=b_{1}$ which belongs to both lattices. The displacements $S(x)-x$ diverge when $x$ goes to infinity. The fractional shear $\Sigma$ performs the same global mapping but only involves bounded displacements.

The property of $\Sigma$ to map $\mathrm{L}$ onto $\mathrm{L}^{\prime}=S(\mathrm{~L})$ is due to the fundamental requirement that $s$ is an irreducible vector of $\mathbf{L}$. In this case, $s=S(s)$ is also an irreducible vector of $\mathrm{L}^{\prime}$ so that $\mathrm{L}$ and $L^{\prime}$ share a 1D sublattice spanned by $s$. We conclude that the fractional shear $\Sigma$ also maps $\mathrm{L}$ onto $\mathrm{L}^{\prime}$ because $\Sigma(x)$ differs from $S(x)$ by Round $[\langle\sigma \mid x\rangle] s$, which is a common lattice vector. Actually $\Sigma$ maps any point $x$ of $\mathbf{L}$ onto the nearest point of $L^{\prime}$ lying on the common lattice line parallel to $s$. Finally, the inverse transformation of $\Sigma$ has the same general form and is given by $\sum^{-1}(x)=\lambda-\operatorname{Frac}[\langle\sigma \mid x\rangle] s$.

It is proved in [4] that such fractional shears are the most simple bounded one-to-one mappings between lattices of equal density which involve a $1 \mathrm{D}$ displacement field.

\section{The double shear condition.}

If $\mathrm{L}_{\mathrm{a}}$ and $\mathrm{L}_{\mathrm{b}}$ are any two lattices of equal density, they cannot in general be related by one unique shear $S=I+|s\rangle\langle\sigma|$, where $s$ should be a common lattice vector: the intersection of 
the two lattices $\mathrm{L}_{\mathrm{a}} \cap \mathrm{L}_{\mathrm{b}}$ usually reduces to the origin $\{0\}$. Even if $\mathrm{L}_{\mathrm{b}}$ is rotated by some rotation $R$, standing for a particular orientation relationship, $\mathrm{L}_{\mathrm{a}}$ and $R \mathrm{~L}_{\mathrm{b}}$ will not, in general, share any $1 D$ sublattice and both lie on a bundle of lattice lines parallel to some lattice vector $s$.

The minimal number of successive shears required to map $\mathrm{L}_{\mathrm{a}}$ onto $R \mathrm{~L}_{\mathrm{b}}$ is well defined. This number depends on the rotation $R$ and was proved in $[1,2]$ to be at most and generically four in $3 \mathrm{D}$ and three in $2 \mathrm{D}$. This property can be easily expected on the basis of dimension arguments. Actually, the set of 3D lattices of given density can be labelled by 8 continuous variables necessary to build all 3 by 3 matrices of unit determinant. On the other hand, a 3D shear with a lattice direction depends on the discrete parameter $s$ and on 2 continuous variables which specify $\sigma$ as a wave vector orthogonal to $s$. Therefore 4 shears are required to account for the 8 parameters in 3D. The same argument suggests that 3 shears are generically required to map a 2D lattice onto another 2D lattice of equal density.

However, for particular pairs of lattices and for particular rotations, only two shears may be enough to map the first lattice onto the second one. In $2 \mathrm{D}$ this double shear condition can always be satısfied provided the lattices have a particular relative orientation which we believe to be physically significant. In this situation, an intermediate $A$ lattice is naturally involved : $\mathrm{L}_{\mathrm{a}}$ and $A$ are related by a first shear of lattice vector $s_{\mathrm{a}}$, which belongs to them. A second shear maps $A$ onto $R \mathrm{~L}_{\mathrm{b}}$ with a shear vector $R s_{\mathrm{b}}$. The corresponding geometry is now investigated in more detail.

\section{Determination of the $2 \mathrm{D}$ shears in the general case.}

Let $\mathrm{L}_{\mathrm{a}}$ and $\mathrm{L}_{\mathrm{b}}$ denote two lattices in $3 \mathrm{D}$ with respective unit cells of volume $\Omega_{\mathrm{a}}$ and $\Omega_{\mathrm{b}}$. We assume that there exist a lattice plane $\mathrm{P}_{\mathrm{a}}$ of $\mathrm{L}_{\mathrm{a}}$ and a lattice plane $\mathrm{P}_{\mathrm{b}}$ of $\mathrm{L}_{\mathrm{b}}$ such that the $2 \mathrm{D}$ lattices $\Lambda_{\mathrm{a}}=\mathrm{L}_{\mathrm{a}} \cap \mathrm{P}_{\mathrm{a}}$ and $\Lambda_{\mathrm{a}}=\mathrm{L}_{\mathrm{b}} \cap \mathrm{P}_{\mathrm{b}}$ have the same density of nodes (their $2 \mathrm{D}$ unit cells have the same area). If $\mathrm{P}_{\mathrm{a}}$ and $\mathrm{P}_{\mathrm{b}}$ are orthogonal to the primitive reciprocal vectors $q_{\mathrm{a}}$ and $q_{\mathrm{b}}$, the equality of the $2 \mathrm{D}$ areas reads $\Omega_{\mathrm{a}}\left|q_{\mathrm{a}}\right|=\Omega_{\mathrm{b}}\left|q_{\mathrm{b}}\right|$. The $2 \mathrm{D}$ shears $S_{\mathrm{a}}$ and $S_{\mathrm{b}}$ will be specified in order that the transformed lattices $S_{\mathrm{a}}\left(\Lambda_{\mathrm{a}}\right)$ and $S_{\mathrm{b}}\left(\Lambda_{\mathrm{b}}\right)$ are related by a simple rotation. In other words, we look for a virtual $2 \mathrm{D}$ intermediate lattice $A$ and a rotation $R$ such that

$$
\Lambda=S_{\mathrm{a}}\left(\Lambda_{\mathrm{a}}\right)=R S_{\mathrm{b}}\left(\Lambda_{\mathrm{b}}\right)
$$

The rotation $R$ is partially settled by the requirement that it transforms $\mathrm{P}_{\mathrm{b}}$ into $\mathrm{P}_{\mathrm{a}}$ so that the $2 \mathrm{D}$ lattices $\Lambda_{\mathrm{a}}$ and $R\left(\Lambda_{\mathrm{b}}\right)$ are coplanar. $R$ will be eventually completely specified by the double shear condition. The shears have the following form

$$
\begin{aligned}
& S_{\mathrm{a}}:=I+\left|s_{\mathrm{a}}\right\rangle\left\langle\sigma_{\mathrm{a}}\right| \\
& S_{\mathrm{b}}:=I+\left|s_{\mathrm{b}}\right\rangle\left\langle\sigma_{\mathrm{b}}\right|
\end{aligned}
$$

where $s_{\mathrm{a}}$ and $s_{\mathrm{b}}$ are irreducible lattice vectors in $\Lambda_{\mathrm{a}}$ and $\Lambda_{\mathrm{b}}$ respectively. The covectors $\sigma_{\mathrm{a}}$ and $\sigma_{\mathrm{b}}$ will specify the invariant planes of the shears.

Since $s_{\mathrm{a}}$ and $s_{\mathrm{b}}$ are invariant by the shears $S_{\mathrm{a}}$ and $S_{\mathrm{b}}$ respectively, $s_{\mathrm{a}}$ and $R s_{\mathrm{b}}$ will belong to the $2 \mathrm{D}$ intermediate lattice $A$. We shall assume that these two vectors actually span this lattice. Now 1 is convenient to use adapted bases of the $2 \mathrm{D}$ lattices, $\left\{s_{\mathrm{a}}, t_{\mathrm{a}}\right\}$ for $A_{\mathrm{a}}$ and $\left\{t_{\mathrm{b}}, s_{\mathrm{b}}\right\}$ for $\Lambda_{\mathrm{b}}$, such that $\left\{s_{\mathrm{a}}, t_{\mathrm{a}}\right\}$ and $\left\{t_{\mathrm{b}}, s_{\mathrm{b}}\right\}$ have the same orientation. After possibly changing $s_{\mathrm{b}}$ into $-s_{\mathrm{b}}$ and $t_{\mathrm{b}}$ into $-t_{\mathrm{b}}$, we can also assume that the basis $\left\{s_{\mathrm{a}}, R s_{\mathrm{b}}\right\}$ has also the same orientation. Consequently $\left\{s_{\mathrm{a}}, R s_{\mathrm{b}}\right\}$ is the image by $S_{\mathrm{a}}$ of some basis of $\Lambda_{\mathrm{a}}$ of the form $\left\{s_{\mathrm{a}}, t_{\mathrm{a}}+m s_{\mathrm{a}}\right\}$, where $m$ is an integer. Similarly, the same basis rotated by $R^{-1},\left\{R^{-1} s_{\mathrm{a}}, s_{\mathrm{b}}\right\}$, is the image by $S_{\mathrm{b}}$ of a basis of $\Lambda_{\mathrm{b}}$ of the form $\left\{t_{\mathrm{b}}+n s_{\mathrm{b}}, s_{\mathrm{b}}\right\}$ (see Fig. 2 where 

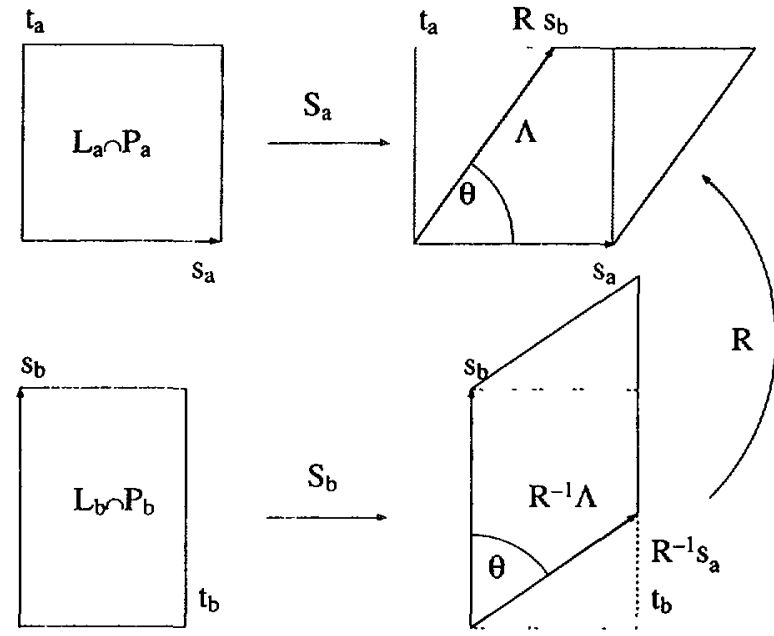

Fig. 2. - The shears $S_{\mathrm{a}}$ and $S_{\mathrm{b}}$ transform the 2D lattices $\mathrm{L}_{\mathrm{a}} \cap \mathrm{P}_{\mathrm{a}}$ and $\mathrm{L}_{\mathrm{b}} \cap \mathrm{P}_{\mathrm{b}}$ into the same 2D lattice $A$, up to a rotation $R$ which specified the orientation relationship. The $2 \mathrm{D}$ intermediate lattice $A$ has a basis $\left\{s_{\mathrm{a}}, R, s_{\mathrm{b}}\right\}$ which is the image of the basis $\left\{s_{\mathrm{a}}, t_{\mathrm{a}}\right\}$ by the shear $S_{\mathrm{a}}$, and of the basis $\left\{s_{\mathrm{b}}, t_{\mathrm{b}}\right\}$ by the shear $S_{\mathrm{b}}$, followed by the rotation $R$.

$m$ and $n$ are assumed to vanish). Different $2 \mathrm{D}$ shears are solutions of this problem but the integers $m$ and $n$ will be chosen in order to get the smallest shear parameters.

With this notation, the $2 \mathrm{D}$ shear transformations read

$$
\begin{aligned}
& S_{\mathrm{a}}\left(s_{\mathrm{a}}\right)=s_{\mathrm{a}} \\
& S_{\mathrm{a}}\left(t_{\mathrm{a}}+m s_{\mathrm{a}}\right)=t_{\mathrm{a}}+\left[\left\langle\sigma_{\mathrm{a}} \mid t_{\mathrm{a}}\right\rangle+m\right] s_{\mathrm{a}} \\
& S_{\mathrm{b}}\left(t_{\mathrm{b}}+n s_{\mathrm{b}}\right)=t_{\mathrm{b}}+\left[\left\langle\sigma_{\mathrm{b}} \mid t_{\mathrm{b}}\right\rangle+n\right] s_{\mathrm{b}} \\
& S_{\mathrm{b}}\left(s_{\mathrm{b}}\right)=s_{\mathrm{b}} .
\end{aligned}
$$

The basis $\left\{s_{\mathrm{a}}, R s_{\mathrm{b}}\right\}$ of the intermediate lattice $A$ is related to $\left\{s_{\mathrm{a}}, t_{\mathrm{a}}\right\}$ and $\left\{t_{\mathrm{b}}, s_{\mathrm{b}}\right\}$ by the following :

$$
\begin{aligned}
& s_{\mathrm{a}}=R S_{\mathrm{b}}\left(t_{\mathrm{b}}+n s_{\mathrm{b}}\right)=R t_{\mathrm{b}}+\left[\left\langle\sigma_{\mathrm{b}} \mid t_{\mathrm{b}}\right\rangle+n\right] R s_{\mathrm{b}} \\
& R s_{\mathrm{b}}=S_{\mathrm{a}}\left(t_{\mathrm{a}}+m s_{\mathrm{a}}\right)=t_{\mathrm{a}}+\left[\left\langle\sigma_{\mathrm{a}} \mid t_{\mathrm{a}}\right\rangle+m\right] s_{\mathrm{a}} .
\end{aligned}
$$

Since the shears have unit determinant we have

$$
s_{\mathrm{a}} \times R s_{\mathrm{b}}=\Omega_{\mathrm{a}} q_{\mathrm{a}}=\Omega_{\mathrm{b}} R q_{\mathrm{b}} .
$$

This cross product specifies the area of the unit cell of $A$, so that the angle $\theta$ between $s_{\mathrm{a}}$ and $R s_{\mathrm{b}}$ can be deduced from

$$
\left|s_{\mathrm{a}}\right|\left|s_{\mathrm{b}}\right| \sin (\theta)= \pm \Omega_{\mathrm{a}}\left|q_{\mathrm{a}}\right|= \pm \Omega_{\mathrm{b}}\left|q_{\mathrm{b}}\right|
$$

A necessary condition for a solution to exist is that $\left|s_{\mathrm{a}}\right|\left|s_{\mathrm{b}}\right| \geqslant \Omega_{\mathrm{a}}\left|q_{\mathrm{a}}\right|$, or equivalently, $\left|s_{\mathrm{a}}\right|\left|s_{\mathrm{b}}\right| \geqslant \Omega_{\mathrm{b}}\left|q_{\mathrm{b}}\right|$. In this case, two possible rotations $R$ are now completely determined by the conditions $R q_{\mathrm{b}}=q_{\mathrm{a}}$ and $\quad\left(s_{\mathrm{a}}, R s_{\mathrm{b}}\right)= \pm \theta$. 
The parameters of the shears can be partially determined by computing the scalar product of the above intermediate basis vectors by $R s_{\mathrm{b}}$ and $s_{\mathrm{a}}$ respectively

$$
\frac{\left|s_{\mathrm{a}}\right|\left|s_{\mathrm{b}}\right| \cos (\theta)-\left(s_{\mathrm{b}}, t_{\mathrm{b}}\right)}{s_{\mathrm{b}}^{2}}=\left\langle\sigma_{\mathrm{b}}, t_{\mathrm{b}}\right\rangle+n
$$

and

$$
\frac{\left|s_{\mathrm{a}}\right|\left|s_{\mathrm{b}}\right| \cos (\theta)-\left(s_{\mathrm{a}}, t_{\mathrm{a}}\right)}{s_{\mathrm{a}}^{2}}=\left\langle\sigma_{\mathrm{a}}, t_{\mathrm{a}}\right\rangle+m .
$$

Different values of $\left\langle\sigma_{\mathrm{a}} \mid t_{\mathrm{a}}\right\rangle$ and $\left\langle\sigma_{\mathrm{b}} \mid t_{\mathrm{b}}\right\rangle$ are obtained depending on $m$ and $n$. They actually give the same geometry so that we may choose the smallest shear parameters

$$
\begin{aligned}
& \left\langle\sigma_{\mathrm{a}} \mid t_{\mathrm{a}}\right\rangle=\operatorname{Frac}\left[\frac{\left|s_{\mathrm{a}}\right|\left|s_{\mathrm{b}}\right| \cos (\theta)-\left(s_{\mathrm{a}}, t_{\mathrm{a}}\right)}{s_{\mathrm{a}}^{2}}\right] \\
& \left\langle\sigma_{\mathrm{b}} \mid t_{\mathrm{b}}\right\rangle=\operatorname{Frac}\left[\frac{\left|s_{\mathrm{a}}\right|\left|s_{\mathrm{b}}\right| \cos (\theta)-\left(s_{\mathrm{b}}, t_{\mathrm{b}}\right)}{s_{\mathrm{b}}^{2}}\right]
\end{aligned}
$$

and

$$
\begin{aligned}
& m=\text { Round }\left[\frac{\left|s_{\mathrm{a}}\right|\left|s_{\mathrm{b}}\right| \cos (\theta)-\left(s_{\mathrm{a}}, t_{\mathrm{a}}\right)}{s_{\mathrm{a}}^{2}}\right] \\
& n=\operatorname{Round}\left[\frac{\left|s_{\mathrm{a}}\right|\left|s_{\mathrm{b}}\right| \cos (\theta)-\left(s_{\mathrm{b}}, t_{\mathrm{b}}\right)}{s_{\mathrm{b}}^{2}}\right] .
\end{aligned}
$$

Finally, we see that the shear parameters are derived by the above formula and only depend on the choice of the shear vectors $s_{\mathrm{a}}$ and $s_{\mathrm{b}}$.

\section{Application to BCC and FCC lattices.}

The previous model is now applied to the 2D lattices provided by the (111) plane in the fcc lattice and the (110) plane of the bcc latt1ce (see Fig. 3). The (111) fcc lattice plane is spanned by $v_{\mathrm{fcc}}=[1 / 2,-1 / 2,0]_{\mathrm{fcc}}$ and $w_{\mathrm{fcc}}=[1 / 2,0,-1 / 2]_{\mathrm{fcc}}$. The unit cell 1 s a rhombus with angles $60^{\circ}$ and $120^{\circ}$. Since the shear vector $s_{\text {fcc }}$ controls the bound on the displacements induced by the fractional shear we shall only consider the two smallest cases (up to symmetry): $s_{\mathrm{fcc}}=v_{\mathrm{fcc}}$ and $s_{\mathrm{fcc}}=v_{\mathrm{fcc}}+w_{\mathrm{fcc}}$. The $(110)_{\mathrm{bcc}}$ lattice plane is spanned by $v_{\mathrm{bcc}}=[1 / 2,-1 / 2,1 / 2]_{\mathrm{bcc}}$ and $w_{\mathrm{bcc}}=[1 / 2,-1 / 2,-1 / 2]_{\mathrm{bcc}}$. The unit cell is a rhombus with angles $\approx 70.53^{\circ}$ and $109.47^{\circ}$. Similarly, we shall assume that the shear vector $s_{\text {bcc }} 1$ of type $v_{\mathrm{bcc}}$ or $v_{\mathrm{bcc}} \pm w_{\mathrm{bcc}}$

The application of the previous model requires that the $2 \mathrm{D}$-lattice has the same density. In other words the unit cell areas must be equal. This condition leads to a simple consequence on the cell parameters : $a_{\mathrm{bcc}} / a_{\mathrm{fcc}}=(3 / 8)^{1 / 4} \approx 0.7825$.

The table I lists the first five non-equivalent solutions of the double shear geometry. Each column gives the principal parameters of the shears. The orientation relationship is characterized in the last three lines by the angle between a simple fcc direction and a simple bcc direction.

Figure $4 \mathrm{a}$ illustrates the solution corresponding to the smallest angle $\left(0.6287^{\circ}\right)$ between $[-2,1,1]_{t c c}$ and $[-1,0,1]_{\mathrm{bcc}}$. Figure $4 \mathrm{~b}$ gives the solution corresponding to the smallest 
Table I.

\begin{tabular}{|c|c|c|c|c|c|}
\hline$\angle\left(v_{\mathrm{FCC}}, \mathrm{v}_{\mathrm{BCC}}\right)$ & -5.8931 & 61.4460 & -1.6619 & -111.5949 & -13.7705 \\
\hline $\mathrm{s}_{\mathrm{FCC}}$ & {$\left[\begin{array}{ll}1-1 & 0\end{array}\right]$} & {$[2-1-1]$} & {$\left[\begin{array}{ll}1-1 & 0\end{array}\right]$} & {$[2-1-1]$} & {$\left[\begin{array}{lll}-1 & 0 & 1\end{array}\right]$} \\
\hline$\sigma_{\mathrm{FCC}}$ & $(0.0,-0.146)$ & $(0039,-0068)$ & $(0.0,-0.321)$ & $(-0.099,0.171)$ & $(0.267,-0.154)$ \\
\hline$\left.<\sigma_{\mathrm{rCC}} \mathrm{v}_{\mathrm{FCC}}\right\rangle$ & 0.0 & 0.0279 & 0.0 & -0.0700 & 0.1890 \\
\hline$\left\langle\sigma_{\mathrm{FCC}}{ } w_{\mathrm{FCC}}>\right.$ & -0.0894 & -0.0279 & -0.1964 & 0.0700 & 0.0 \\
\hline $\mathrm{s}_{\mathrm{BCC}}$ & {$[1-1-1]$} & {$\left[\begin{array}{ll}1 & 1-1\end{array}\right]$} & 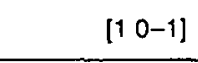 & {$\left[\begin{array}{lll}0 & 1 & 0\end{array}\right]$} & {$\left[\begin{array}{lll}0 & 1 & 0\end{array}\right]$} \\
\hline$\sigma_{\mathrm{BCC}}$ & $(0.168,-0.059)$ & $(00,0.195)$ & $(0.048,-0.067)$ & $(0.153,0.108)$ & $(-0.092,-0.065)$ \\
\hline$<\left.\sigma_{\mathrm{BCC}}\right|^{\gamma_{\mathrm{BCC}}}>$ & 0.1136 & 0.0 & 0.0322 & 0.1038 & -0.0626 \\
\hline$<\sigma_{\mathrm{FCC}} \mid w_{\mathrm{BCC}}>$ & 0.0 & 01249 & -0.0322 & 0.1038 & -0.0626 \\
\hline $\mathrm{u}_{\mathrm{FCC}}$ & {$\left[\begin{array}{lll}-2 & 1 & 1\end{array}\right]$} & {$\left[\begin{array}{lll}-1 & 0 & 1\end{array}\right]$} & {$\left[\begin{array}{lll}-1 & 1 & 0\end{array}\right]$} & {$\left[\begin{array}{lll}-1 & 1 & 0\end{array}\right]$} & {$\left[\begin{array}{lll}-1 & 0 & 1\end{array}\right]$} \\
\hline $\mathrm{u}_{\mathrm{BCC}}$ & {$\left[\begin{array}{lll}-1 & 0 & 1\end{array}\right]$} & {$\left[\begin{array}{lll}-1-1 & 1\end{array}\right]$} & {$\left[\begin{array}{lll}-1-1 & 1\end{array}\right]$} & {$[1-1-1]$} & {$\left[\begin{array}{lll}-1 & 1 & 1\end{array}\right]$} \\
\hline$\angle\left(\mathrm{u}_{\mathrm{FCC}}, \mathrm{u}_{\mathrm{BCC}}\right)$ & 0.6287 & 1.4460 & 1.6679 & 2.1237 & 3.2417 \\
\hline
\end{tabular}

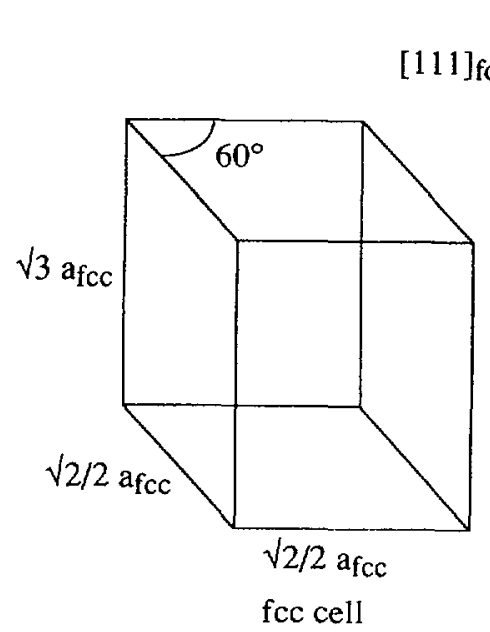

$[110]_{\mathrm{bcc}}$

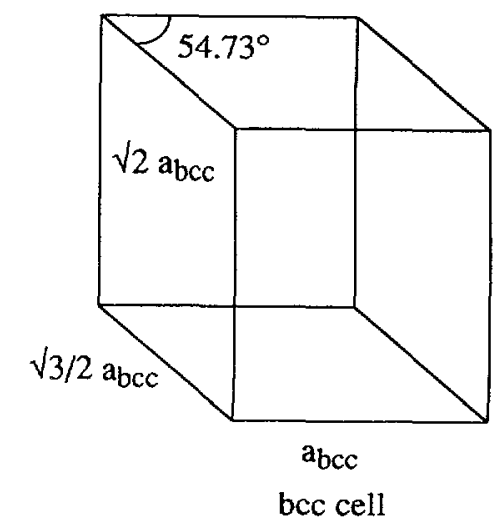

Fig. 3. - The unit cells of the fcc lattice and of the bcc lattice. The interface plane is $\left[\begin{array}{lll}1 & 1 & 1\end{array}\right]_{\mathrm{fcc}}=\left[\begin{array}{lll}1 & 10\end{array}\right]_{\mathrm{bcc}}$. In the case of the duplex steel under consideration, the areas of the $2 \mathrm{D}$ unit cells in the common latt1ce plane are equal.

angle $\left(1.4460^{\circ}\right)$ between $[-1,0,1]_{\mathrm{fcc}}$ and $[-1,-1,1]_{\mathrm{bcc}}$. The displacement field indicated in figures 4 results from the combination of the two fractional shears.

This particular case exemplifies clearly how the present model realızes a global transformation of the lattice by site displacement on the microscopic scale. The only requirement of the 


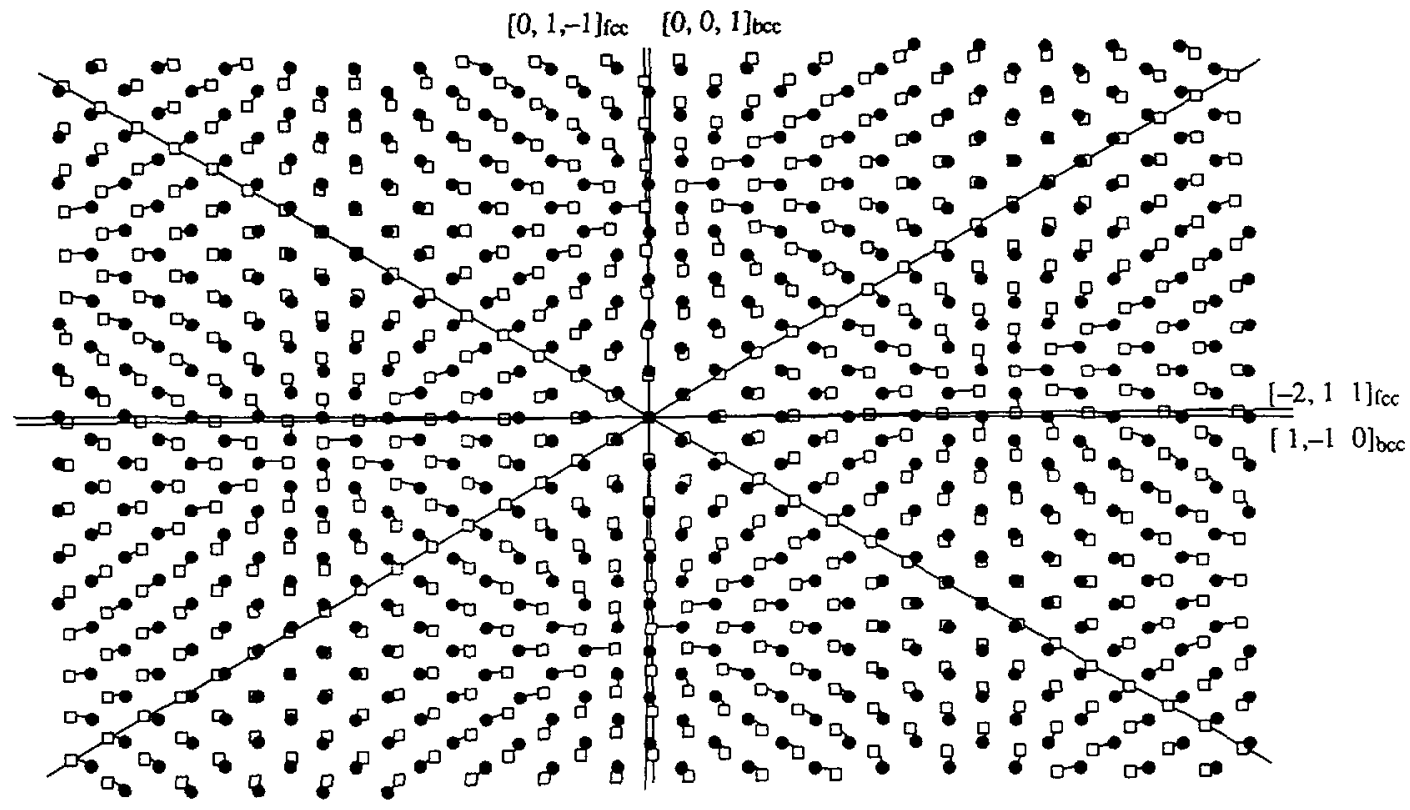

a)

$[-1,-1,1]_{\mathrm{bcc}}[-1,0,1]_{\mathrm{fcc}}$

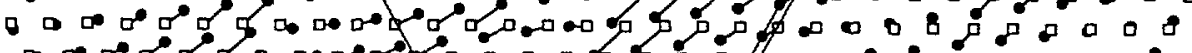

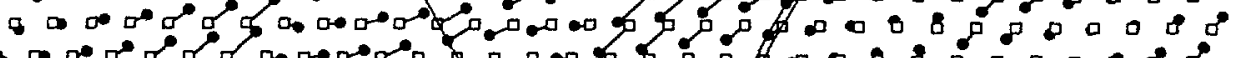

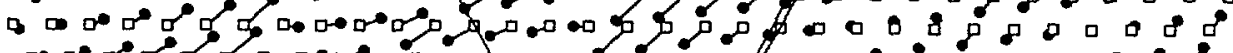
0

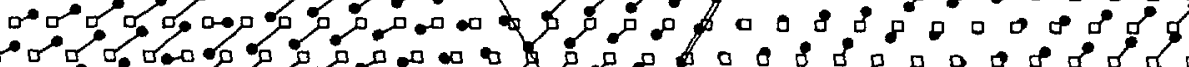

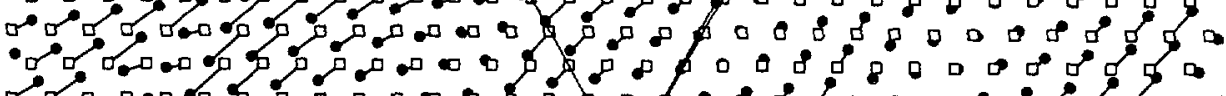

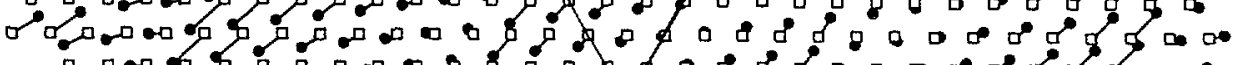

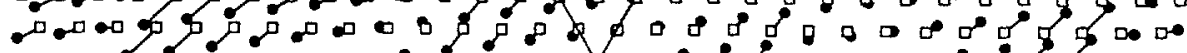

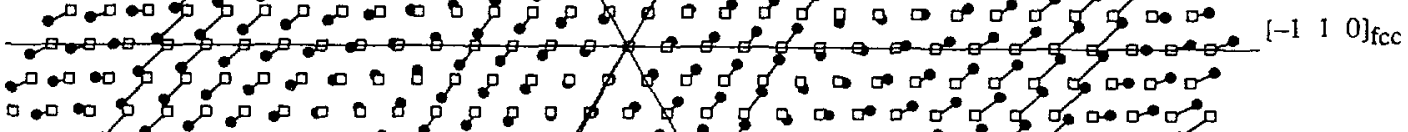

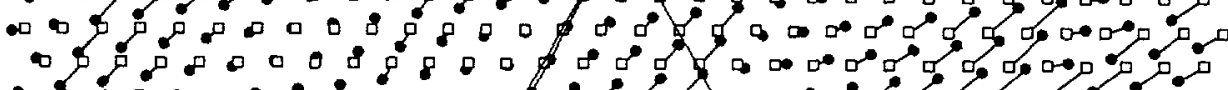
०

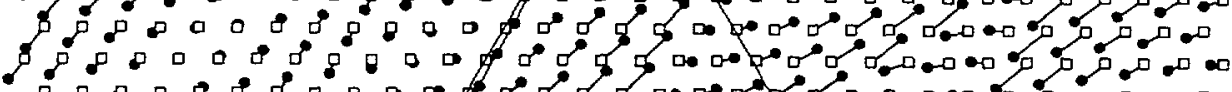
50

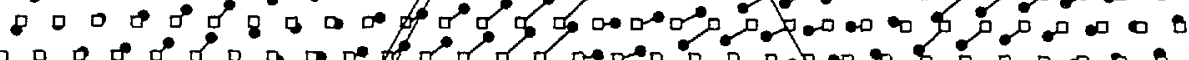

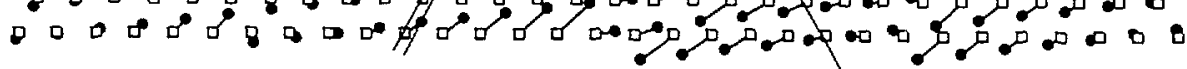

b)

Fig. 4. - White squares belong to the (111) fsc plane and black circles belong to the (1111) bce plane. a) The shear vectors are $[1-10]_{\mathrm{fcc}}$ and $[1-1-1]_{\mathrm{bcc}}$. The relatıve orientation is specified by the angle $0.6287^{\circ}$ between $[-211]_{\mathrm{fcc}}$ and $[-101]_{\mathrm{bcc}}$. This solution is close to $\mathrm{N}-\mathrm{W}$ orientation relationship. b) The shear vectors are $[2-1-1]_{\mathrm{fcc}}$ and $[11-1]_{\mathrm{bcc}}$ and the relative orientation is specified by the angle $14460^{\circ}$ between $[-101]_{\mathrm{fcc}}$ and $[-1-11]_{\mathrm{bcc}}$ which is close to K-S. 
$2 \mathrm{D}$ model is that the planar site density is unchanged. Therefore this model is a reasonable candidate to account for displacive transformations such as the martensitic transformation. Martensitic transformations are non-diffusive lattice transformations commonly encountered in metallurgy (for a review see [5]). The most classical one concerns the iron system in which austenite, solid solution of fcc iron transforms without diffusion in a centered tetragonal crystal close to ferrite $(c / a=1.05)$.

It is remarkable that the sets of orientation relationships listed in table I are reminiscent of those martensite relating to its parent phase : the first column in table I recalls the NishiyamaWasserman (N-W) orientation relationship, while the other solutions are closer to the Kurdjumov-Sachs (K-S) case. We recall that both experimental orientations set the $(111)_{\mathrm{fcc}}$ plane parallel to the $(110)_{\mathrm{bcc}}$ plane but in the K-S case : $[1-110]_{\mathrm{fcc}} / /[1-11]_{\mathrm{bcc}}$ when with the N-W relation: $[-211]_{f c c} / /[01-1]_{b c c}$ (equivalent to $[10-1]_{f c c} / /[001]_{b c c}$ ).

Finally it should be noted that the conditions on the equality of lattice density provide a very simple criterion for the cell parameters of both lattices. For example, as far as bcc and fcc lattices are concerned, a 3D double shear mechanism requires equal volumes: i.e. $a_{\mathrm{bcc}} / a_{\mathrm{fcc}}=2^{-1 / 3}(=0.7937)$. A $1 \mathrm{D}$ mechanism needs equal interplanar spacing between some planes of both lattices. In other words each mechanism is characterized by a particular cell parameter ratio. Incidentally we do not expect that the $2 \mathrm{D}$ model is relevant for all the martensitic transformations but only for some cases.

The following discussion will focus on the predictions deriving of the $2 \mathrm{D}$ version of the double shear mechanism and the features encountered in some metallurgical cases.

\section{Discussion.}

A martensitic transformation is characterized by two main features : the orientation relationships and the interface plane (usually called habit plane) between both phases. As mentioned previously the model predicts orientation relationships which are close to those encountered in steels. A habit plane is also predicted by our model : in the $2 \mathrm{D}$ restriction of the double shear mechanısm the habit plane is the transformation plane. For the fcc/bcc transformation previously achieved, the habit plane is the $(111)_{\text {fcc }}$ plane $\left.(=110)_{b c c}\right)$.

Experımentally, this habit plane is encountered (for example in low carbon steels) but not as a general case (habit planes oriented $(225)_{\mathrm{fcc}}$ and $(259)_{\mathrm{fcc}}$ are frequently observed [6]. Anyhow these habit planes are not the most relevant for comparison with our model since the product of the martensitic transformation is martensite, a centered tetragonal structure, not ferrite, the body centered cubic crystal. A bibliographical survey has brought to our knowledge duplex steels which seem to justify the present model.

Duplex steels consist in a two-phase microstructure: ferrite (bcc) labelled $\alpha$ or $\delta$, and austenite ( $\mathrm{fcc}$ ) labelled $\gamma$. They provide a wide spectrum of structures : from dispersion of small austenite grans in a ferritic matrix to the opposite case of ferrite grains in austenite. These particular microstructure are obtained owing to appropriate high temperature heat treatments $\left(600^{\circ} \mathrm{C}\right.$ to $1300^{\circ} \mathrm{C}$ ) and to the addition of numerous solute elements $(\mathrm{Ni}, \mathrm{Cr}, \mathrm{Mo}$, $\mathrm{Mn}, \mathrm{N} .$. ). At room temperature these solute elements are shared unequally between both phases (for an introduction to duplex steels see [7]). The two-phase microstructure results from martensitic transformation as well as diffusion. The martensitic stage is revealed by the existence of the K-S or N-W orientation relationships between the austenite and ferrite with $(111)_{\text {fcc }}$ habit plane. These typical features are encountered in two frequent microstructures of duplex steels: the growth of austenite particles within ferrite grains and the Widmanstätten ferrite laths.

In the former microstructure, Transmission Electron Microscopy and in-situ chemical analysis have revealed the typical martensitic morphology of the austenite platelets (onientation 
relationships and habit planes) but that the chemical composition of the austenite particles might differ or not from the one of the ferrite matrıx $([8,9]$. Widmanstätten laths consist of side plates of ferrite nucleating at the austenite grain boundaries but growing in the matrix among well defined planes $\left((111)_{\mathrm{fcc}}\right.$ plane in duplex steels $\left.[7,8,10]\right)$.

Though these duplex steels provide microstructures in good agreement with our 2D model, it is not possible to test with accuracy whether they obey the primary requirement of our approach : a constant site density in the transformation plane. As mentioned previously, this condition is expressed by a particular cell parameter ratio. Unfortunately cell parameters are known only at room temperature and their values at high temperatures are not only modified by dilation but also by the partition of a large amount of solute elements between austenite and ferrite, solute elements which are known to modify the cell parameters [11, 12]. Since, to our knowledge, no high temperature cell parameters have been measured, the evolution of the cell parameter ratio with temperature is badly known. Taking only the dilation effect into account, figure 5 compares the evolution with temperature of the cell parameter ratio in a duplex steel $\left(\mathrm{CF} 8 \mathrm{M}, a_{\mathrm{bcc}}=0.285 \mathrm{~nm}, a_{\mathrm{fcc}}=0.3598 \mathrm{~nm}\right.$ [13]) with some remarkable values relative to the site density conservation. The usual dilation coefficients of austenite and ferrite have been here used (Austenite : $\alpha_{\mathrm{fcc}}=23 \times 10^{-6} \mathrm{~K}^{-1}$, Ferrite : $\alpha_{\mathrm{bcc}}=14 \times 10^{-6} \mathrm{~K}^{-1}$ ). At high temperature the cell parameter ratio tends to meet the value required by the present $2 \mathrm{D}$ model. This is in agreement with the necessity of high temperature treatment to develop the previously mentioned microstructures. However because of the unknown compositional effect the comparison with experimental data should not be further developed.

Actually the point is that our requirement on cell parameter ratio meets well known features of the martensitic transformation : the relation between the cell parameter ratio, habit planes and orientation relationships. This dependence, earlier noticed in carbon steels [6], has been emphasized by a large survey of the experimental data [14]. Such a phenomenon is interpreted with the metallurgical models in use to describe the martensitic transformation. These models, which are first all macroscopic, are based on the assumption that the martensitic transformation involves shears and keeps invariant some atomic line. Therefore, after a shear deformation,

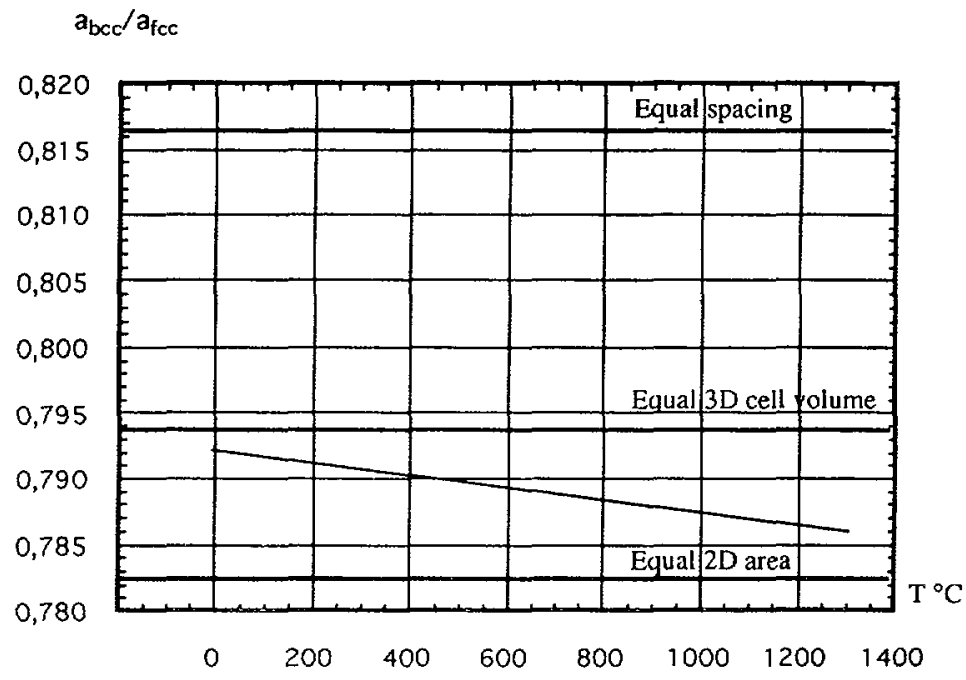

Fig. 5. - The dependence of the lattice parameters for a duplex steel (CF8M) [13] as a function of temperature. The horizontal lines specify the three different ratios $a_{\mathrm{fcc}} / a_{\mathrm{bcc}}$ corresponding to the equality of atomic volumes, planar spacing along [ 1111$]_{\mathrm{fc}} / /\left[\begin{array}{ll}1 & 10\end{array}\right]_{\mathrm{bcc}}$ or $2 \mathrm{D}$ cell areas. For the CF8M duplex steel, the room temperature data are $a_{\mathrm{bi}}=0.285 \mathrm{~nm}, a_{\mathrm{ic}}=0.3598 \mathrm{~nm}$ ). 
some rigid body rotation generating a particular orientation relationship is necessary to get an invariant line. This approach is phenomenological since the invariant line hypothesis is only derived from the observations. Our model also predicts a strong dependence of orientation relationship and habit planes since the mechanism will depend on the site density. The strength point is that, in our work, this dependence is derived from the most basic principle: the conservation of the lattıce density. This very conservation indicates which shear mechanism is «mathematically » allowed: $1 \mathrm{D}, 2 \mathrm{D}$ or $3 \mathrm{D}$.

\section{Conclusion.}

To our knowledge the present model, though restricted to the 2-dimensional case, is the first to achieve a global as well as a microscopic transformation which, when applied to the fcc/bcc lattices predicts the observed orientation relationships. It is worth noting that the experimentally noticed dependence of the mechanism on the cell parameter ratio is also accounted for by our approach. Because of the present $2 \mathrm{D}$ restriction, the model cannot be considered as relevant for the most general case of transformation, still a class of materials (the duplex steels) provides experimental features in this $2 \mathrm{D}$ frame.

In the present model the site displacement results from the combination of two shear transformations. This double shear is sufficient to generate the observed orientation relationship between the lattices. This remarkable property is one of the most general ones of the transformation between the lattıces of equal density. Therefore the double shear mechanism appears as the most natural basis for displacive transformations, however it should be stressed that a fractional double shear should be used in order to have physically meaningful site displacements.

As mentioned in the discussion the observed (225), (259) habit planes cannot be supported by the present $2 \mathrm{D}$ approach. In general this $2 \mathrm{D}$-model is not likely to be relevant for all martensitic transformation but only in those cases where some lattice planes of both phases have the same density of nodes. Therefore with the same basic ideas (fractional double shears), we will examine the $1 \mathrm{D}$ on $3 \mathrm{D}$ cases further on : i.e. lattices exhibiting etther a direction with constant atomic spacing or a constant atomic volume.

\section{References}

[1] Duneau M. and Oguey C., J. Phys. France 51 (1990) 5-19.

[2] Duneau M. and Oguey C., J. Phys. A. (1991) 24 461-475.

[3] Wayman C. M., Introduction to the crystallography of martensitic transformation (Mac Millan, New York, 1964).

[4] Duneau M., J. Phys. I France (1992) 871-886.

[5] Christian J. W., The Theory of Transformations in Metals and Alloys (Pergamon Press, Oxford, Edward Arnold Publishers, 1965).

[6] Crocker A. G. and Bilby B. A., Acta Met. 9 (1961) 678.

[7] Honeycombe R. W. K., Steels : microstructures and properties (1981).

[8] Redjaïmia A., Metauer G., Gantois M., Proc. Conf. on Duplex Stannless Steels, DSS'91, Beaume, France, J. Charles and S. Bernhardsson Eds. (Edition de physique), Vol. 1 (1931) pp. 119-126.

[9] Chance J., Coop W ;, Gradwell K. J., Conf. Proceeding, Duplex Stanless Steels, R. U. Lula Ed. (ASM Metals Park, Ohio 44073, 1978, 371.

[10] Redjaimia A., Thesis of the University of Nancy (1991)

[11] Pickering F. B., Physical metallurgy and the design of steels (Applied science publishers Ltd, London, 1978).

[12] Sutton A. L. and Hume-Rothery W., Philos. Mag. 46 (1955) 1295.

[13] Sidhom H., Thesis of the university of Paris XI, 1990.

[14] Dahmen U., Acta Met. 30 (1982) 63. 